\title{
Local Equivalence of Rank-Two Quantum Mixed States
}

\author{
Sergio Albeverio ${ }^{a}{ }^{1}$, Shao-Ming $\mathrm{Fei}^{b, c}$, Debashish Goswami ${ }^{d}{ }^{2}$ \\ ${ }^{a}$ Institut für Angewandte Mathematik, Universität Bonn, D-53115 \\ ${ }^{b}$ Department of Mathematics, Capital Normal University, Beijing 100037 \\ ${ }^{c}$ Max Planck Institute for Mathematics in the Sciences, D-04103 Leipzig \\ ${ }^{d}$ Statistics and Mathematics Unit, Indian Statistical Institute, Kolkata 700108
}

\begin{abstract}
We investigate the equivalence of quantum mixed states under local unitary transformations. For a class of rank-two mixed states, a sufficient and necessary condition of local equivalence is obtained by giving a complete set of invariants under local unitary transformations, such that two states in this class are locally equivalent if and only if all these invariants have equal values for them.
\end{abstract}

PACS numbers: 03.67.-a, 02.20.Hj, 03.65.-w

Quantum entanglement has been extensively investigated as a key physical resource to realize quantum information tasks such as quantum cryptography, quantum teleportation and quantum computation [1]. Due the fact that the properties of entanglement for multipartite quantum systems remain invariant under local unitary transformations on the subsystems, the entanglement can be characterized in principle by all the invariants under local unitary transformations. For instance, the trace norms of realigned or partial transposed density matrices in entanglement measure and separability criteria are some of these invariants [2]. Therefore a complete set of invariants gives rise to the classification of the quantum states under local unitary transformations. Two quantum states are locally equivalent if and only if all these invariants have equal values for these states.

There have been many results on calculation of invariants $[3,4]$ related to the equivalence of quantum states under local unitary transformations, e.g. for general two-qubit systems [5], three-qubit states [6,7], some generic mixed states [8, 9, 10], some classes of tripartite pure and mixed states [11]. However till now we still have no operational criteria to judge

\footnotetext{
${ }^{1}$ SFB 611; BiBoS; IZKS; CERFIM(Locarno); Acc. Arch.; USI(Mendrisio)

${ }^{2}$ research partially supported by Av Humboldt Foundation
} 
the equivalence for two general bipartite mixed states under local unitary transformations. In this letter we investigate the local equivalence under local unitary transformations for a class of rank-two bipartite mixed quantum states in arbitrary dimensions, and present an operational criterion.

Let $H_{1}$ and $H_{2}$ be $m$ and $n$-dimensional complex Hilbert spaces, with $\left|e_{\alpha}\right\rangle, \alpha=1, \ldots, m$, and $\left|f_{\beta}\right\rangle, \beta=1, \ldots, n, m \leq n$, as orthonormal bases. Let $\rho_{1}$ and $\rho_{2}$ be two bipartite density matrices defined on $H_{1} \otimes H_{2}$ with rank $r\left(\rho_{1}\right)=r\left(\rho_{2}\right)=2$. $\rho_{1}$ and $\rho_{2}$ are said to be equivalent under local unitary transformations if there exist unitary operators $U_{1}$ on $H_{1}$ and $U_{2}$ on $H_{2}$ such that

$$
\rho_{2}=\left(U_{1} \otimes U_{2}\right) \rho_{1}\left(U_{1} \otimes U_{2}\right)^{\dagger},
$$

where $\dagger$ stands for transpose and conjugation.

As $\rho_{1}$ and $\rho_{2}$ are rank-two density matrices, they have the following decompositions according to their eigenvalues and eigenvectors:

$$
\rho_{i}=\sum_{\alpha=1}^{2} \lambda_{\alpha}^{i}\left|\nu_{\alpha}^{i}\right\rangle\left\langle\nu_{\alpha}^{i}\right|, \quad i=1,2,
$$

where $\lambda_{\alpha}^{i}$ and $\left|\nu_{\alpha}^{i}\right\rangle, \alpha=1,2$, are the nonzero eigenvalues and eigenvectors of the density matrix $\rho^{i}$ respectively, $\sum_{\alpha=1}^{2} \lambda_{\alpha}^{i}=1$. $\left|\nu_{\alpha}^{i}\right\rangle$ has generally the form

$$
\left|\nu_{1}^{i}\right\rangle=\sum_{\alpha=1}^{m} \sum_{\beta=1}^{n} a_{\alpha \beta}^{i}\left|e_{\alpha}\right\rangle \otimes\left|f_{\beta}\right\rangle, \quad\left|\nu_{2}^{i}\right\rangle=\sum_{\alpha=1}^{m} \sum_{\beta=1}^{n} b_{\alpha \beta}^{i}\left|e_{\alpha}\right\rangle \otimes\left|f_{\beta}\right\rangle,
$$

where $a_{\alpha \beta}^{i}, b_{\alpha \beta}^{i} \in \mathbb{C}, \sum_{\alpha \beta} a_{\alpha \beta}^{i} a_{\alpha \beta}^{i *}=\sum_{\alpha \beta} b_{\alpha \beta}^{i} b_{\alpha \beta}^{i *}=1, i=1,2$, * denotes complex conjugation.

Let $A_{i}$ and $B_{i}$ denote the $m \times n$ matrices with entries $a_{\alpha \beta}^{(i)}$ and $b_{\alpha \beta}^{(i)}$ respectively. We consider the necessary and sufficient conditions of equivalence under local unitary transformations for a class of rank-two states satisfying the following conditions:

$$
A_{i}^{\dagger} A_{i}=B_{i}^{\dagger} B_{i}, \quad A_{i} A_{i}^{\dagger}=B_{i} B_{i}^{\dagger} \quad \text { for } i=1,2 .
$$

[Theorem] The density matrices $\rho_{1}$ and $\rho_{2}$ are equivalent under local unitary transformations if and only if the following hold:

(i) $\operatorname{Tr}\left(\rho_{1}^{2}\right)=\operatorname{Tr}\left(\rho_{2}^{2}\right)$;

(ii) $\operatorname{Tr}\left(\left(A_{1} B_{1}^{\dagger}\right)^{\alpha}\right)=\operatorname{Tr}\left(\left(A_{2} B_{2}^{\dagger}\right)^{\alpha}\right), \forall \alpha=1, \ldots, m$;

(iii) $r\left(A_{1}\right)=r\left(A_{2}\right), r\left(B_{1}\right)=r\left(B_{2}\right), r\left(\left(B_{1}^{\dagger} A_{1}\right)^{\alpha}\right)=r\left(\left(B_{2}^{\dagger} A_{2}\right)^{\alpha}\right), \forall \alpha=1, \ldots, m$.

[Proof] It is straightforward to see that (i)-(iii) above hold if $\rho_{1}$ and $\rho_{2}$ are equivalent under local unitary transformations, in the sense of eq.(1).

We prove the converse. Two pairs of $(m \times n)$ matrices, $(A, B)$ and $(C, D)$, are called contragrediently equivalent if $A=S C T^{-1}, B=T D S^{-1}$ for some invertible matrices $S$ 
and $T$. It is shown in [12] that the pairs $(A, B)$ and $(C, D)$ are contragrediently equivalent if and only if $A B$ is similar to $C D$ and $r(A)=r(C), r(B)=r(D), r(B A)^{\alpha}=r(D C)^{\alpha}$, $r(A B)^{\alpha}=r(C D)^{\alpha}$ for all $\alpha=1, \ldots, m$.

Therefore from the conditions (ii) and (iii) we have that the pairs $\left(A_{1}, B_{1}^{\dagger}\right)$ and $\left(A_{2}, B_{2}^{\dagger}\right)$ are contragrediently equivalent and there are invertible (but not necessarily unitary) matrices $S$ and $T$ such that

$$
S A_{2}=A_{1} T, \quad T B_{2}^{-1}=B_{1}^{-1} S
$$

Eq. (3) can be rewritten as,

$$
\left(\begin{array}{cc}
0 & T \\
S & 0
\end{array}\right)\left(\begin{array}{cc}
0 & A_{2} \\
B_{2}^{\dagger} & 0
\end{array}\right)=\left(\begin{array}{cc}
0 & B_{1}^{\dagger} \\
A_{1} & 0
\end{array}\right)\left(\begin{array}{cc}
0 & T \\
S & 0
\end{array}\right)
$$

By assumption (2), the matrices $W_{1}:=\left(\begin{array}{cc}0 & A_{2} \\ B_{2}^{\dagger} & 0\end{array}\right)$ and $W_{2}:=\left(\begin{array}{cc}0 & B_{1}^{\dagger} \\ A_{1} & 0\end{array}\right)$ are normal.

If two normal matrices $M, N$ and an invertible matrix $X$ satisfy $X M X^{-1}=N$, then one has $U_{X} M U_{X}^{\dagger}=N$, where $X=U_{X}|X|$ is the polar decomposition of $X$ and $U_{X}$ is unitary [13]. Therefore from the observation that the unitary part of the polar decomposition in $\left(\begin{array}{cc}0 & S \\ T & 0\end{array}\right)$ is nothing but $\left(\begin{array}{cc}0 & U_{S} \\ U_{T} & 0\end{array}\right)$, we have

$$
\left(\begin{array}{cc}
0 & U_{T} \\
U_{S} & 0
\end{array}\right)\left(\begin{array}{cc}
0 & A_{2} \\
B_{2}^{\dagger} & 0
\end{array}\right)=\left(\begin{array}{cc}
0 & B_{1}^{\dagger} \\
A_{1} & 0
\end{array}\right)\left(\begin{array}{cc}
0 & U_{T} \\
U_{S} & 0
\end{array}\right)
$$

which is equivalent to

$$
A_{2}=U_{S}^{\dagger} A_{1} U_{T}, \quad B_{2}=U_{S}^{\dagger} B_{1} U_{T}
$$

Here $U_{S}$ and $U_{T}$ are unitary (as $S, T$ are invertible). The condition (i) and $\operatorname{Tr}\left(\rho_{1}\right)=$ $\operatorname{Tr}\left(\rho_{2}\right)=1$ together imply that the density matrices $\rho_{1}$ and $\rho_{2}$ have the same eigenvalues. Therefore $\rho_{2}=\left(U_{1} \otimes U_{2}\right) \rho_{1}\left(U_{1} \otimes U_{2}\right)^{\dagger}$, where $U_{1}=U_{S}^{\dagger}, U_{2}=\left(U_{T}\right)^{t}$ ( $t$ denoting transpose).

The Theorem gives a sufficient and necessary condition for local equivalence of two ranktwo mixed states satisfying (2). The class of quantum states satisfying (2) is not trivial. As a simple example, we consider the two-qubit systems. In this case $A$ and $B$ are $2 \times 2$ matrices. It is easily verified that the following matrices satisfy the required conditions,

$$
A(\theta)=\frac{1}{\sqrt{2}}\left(\begin{array}{cc}
\cos \theta & \sin \theta \\
-\sin \theta & \cos \theta
\end{array}\right), \quad B(\gamma)=\frac{1}{\sqrt{2}}\left(\begin{array}{cc}
\cos \gamma & \sin \gamma \\
\sin \gamma & -\cos \gamma
\end{array}\right)
$$

Hence the rank-two density matrix $\rho=\lambda|\psi\rangle\langle\psi|+(1-\lambda)| \phi\rangle\langle\phi|$, where $|\psi\rangle=\sum_{\alpha, \beta=1}^{2} a_{\alpha \beta}(\theta)\left|e_{\alpha}\right\rangle \otimes$ $\left|f_{\beta}\right\rangle,|\phi\rangle=\sum_{\alpha, \beta=1}^{2} b_{\alpha \beta}(\gamma)\left|e_{\alpha}\right\rangle \otimes\left|f_{\beta}\right\rangle$, belongs to the class we are concerning. From the theorem we have that $\rho$ and $\rho^{\prime}=\lambda\left|\psi^{\prime}\right\rangle\left\langle\psi^{\prime}|+(1-\lambda)| \phi^{\prime}\right\rangle\left\langle\phi^{\prime}\right|$ with $\left|\psi^{\prime}\right\rangle=\sum_{\alpha, \beta=1}^{2} a_{\alpha \beta}\left(\theta^{\prime}\right)\left|e_{\alpha}\right\rangle \otimes\left|f_{\beta}\right\rangle$, $\left|\phi^{\prime}\right\rangle=\sum_{\alpha, \beta=1}^{2} b_{\alpha \beta}\left(\gamma^{\prime}\right)\left|e_{\alpha}\right\rangle \otimes\left|f_{\beta}\right\rangle$, are equivalent under local unitary transformations. 
Here the concurrence $C(|\psi\rangle)=C(|\phi\rangle)=1$. Both pure states $|\psi\rangle$ and $|\phi\rangle$ are maximally entangled. In the special case $\theta=0$ (resp. $\gamma=0$ ), $|\psi\rangle$ (resp. $|\phi\rangle$ ) is reduced to one of the Bell bases $|\psi\rangle=(|00\rangle+|11\rangle) / \sqrt{2}$ (resp. $|\phi\rangle=(|00\rangle-|11\rangle) / \sqrt{2})$. These states are equivalent under local unitary transformations. Nevertheless, generally $\rho$ and $\rho^{\prime}$ are not equivalent under local unitary transformations even if $|\psi\rangle$ (resp. $|\phi\rangle$ ) is equivalent to $\left|\psi^{\prime}\right\rangle$ (resp. $\left|\phi^{\prime}\right\rangle$ ) under local unitary transformations, unless the same local unitary transformations transform $|\psi\rangle$ to $\left|\psi^{\prime}\right\rangle$ and $|\phi\rangle$ to $\left|\phi^{\prime}\right\rangle$ simultaneously.

Generally a rank-two state can be written as $\rho=\lambda\left|\nu_{1}\right\rangle\left\langle\nu_{1}|+(1-\lambda)| \nu_{2}\right\rangle\left\langle\nu_{2}\right|, 0<\lambda<$ 1. The normalized vectors $\left|\nu_{1}\right\rangle$ and $\left|\nu_{2}\right\rangle$ are given by the $m \times n$ matrices $(A)_{\alpha \beta}=a_{\alpha \beta}$ and $(B)_{\alpha \beta}=b_{\alpha \beta}$ respectively, $\left|\nu_{1}\right\rangle=\sum_{\alpha \beta} a_{\alpha \beta}\left|e_{\alpha}\right\rangle \otimes\left|f_{\beta}\right\rangle,\left|\nu_{2}\right\rangle=\sum_{\alpha \beta} b_{\alpha \beta}\left|e_{\alpha}\right\rangle \otimes\left|f_{\beta}\right\rangle$, with $\operatorname{Tr}\left(A A^{\dagger}\right)=\operatorname{Tr}\left(B B^{\dagger}\right)=1$ due to normalization. Let us consider the general forms of a pair of matrices $A$ and $B$ such that the conditions $A^{\dagger} A=B B^{\dagger}$ and $A A^{\dagger}=B B^{\dagger}$ are satisfied.

Since $A^{\dagger} A=B^{\dagger} B$, we can write down singular value decomposition of $A$ and $B$ as follows:

$$
A=U \Delta V^{\dagger}, \quad B=U^{\prime} \Delta V^{\prime \dagger},
$$

where $U, U^{\prime}$ and $V, V^{\prime}$ are unitary matrices and $\Delta$ is a diagonal matrix with nonnegative entries. Furthermore, the condition $A^{\dagger} A=B^{\dagger} B$ implies $V^{\prime \dagger} V \Delta^{2}=\Delta^{2} V^{\prime \dagger} V$. Thus, $V^{\prime \dagger} V$ commutes with $\Delta$. Similarly from $A A^{\dagger}=B B^{\dagger}$ we conclude that $U^{\prime \dagger} U$ also commutes with $\Delta$. Hence we have

$$
B=U^{\prime} \Delta V^{\prime \dagger}=U U^{\dagger} U^{\prime} \Delta V^{\prime \dagger} V V^{\dagger}=U \Gamma \Delta V^{\dagger},
$$

where $\Gamma=\left(U^{\prime \dagger} U\right)^{\dagger} V^{\prime \dagger} V$ is unitary and commutes with $\Delta$. Therefore the pair $(A, B)$ can be transformed into the pair $(\Delta, \Gamma \Delta)$. We call $(\Delta, \Gamma \Delta)$ the canonical form of the pair $(A, B)$. If the diagonal matrix $\Delta$ is of the form $\operatorname{diag}\left(d_{1}, \ldots, d_{1}, d_{2}, \ldots, d_{2}, \ldots, d_{k}, \ldots, d_{k}\right)$, where $d_{i}$ is repeated with multiplicity $m_{i}$, then $\Gamma$ must have the block diagonal form $\operatorname{diag}\left(\Gamma_{1}, \ldots, \Gamma_{k}\right)$, where $\Gamma_{i}, i=1, \ldots, k$, are $m_{i} \times m_{i}$ unitary matrices.

In fact, if we have another pair of matrices $A^{\prime}$ and $B^{\prime}$, associated with the eigenvectors $\left|\nu_{1}^{\prime}\right\rangle$ and $\left|\nu_{2}^{\prime}\right\rangle$ of another rank-two density matrix in the class considered, with canonical form $\left(\Delta^{\prime}, \Gamma^{\prime} \Delta^{\prime}\right)$, then $\left|\nu_{1}^{\prime}\right\rangle,\left|\nu_{2}^{\prime}\right\rangle$ and $\left|\nu_{1}\right\rangle,\left|\nu_{2}\right\rangle$ are equivalent under local unitary transformations if and only if $\Delta=\Delta^{\prime}$ and $w_{i} \Gamma_{i} w_{i}^{\dagger}=\Gamma_{i}^{\prime}$ for some unitary matrix $w_{i}, i=1, \ldots, k$.

Therefore under the local unitary transformation $\rho \rightarrow\left(U \otimes V^{*}\right) \rho\left(U \otimes V^{*}\right)^{\dagger}$, a rank-two mixed state in our class has the standard form: $\rho=\lambda\left|\mu_{1}\right\rangle\left\langle\mu_{1}|+(1-\lambda)| \mu_{2}\right\rangle\left\langle\mu_{2}\right|$, where $\left|\mu_{1}\right\rangle=$ $\sum_{\alpha} d_{\alpha}\left|e_{\alpha}\right\rangle \otimes\left|f_{\alpha}\right\rangle,\left|\mu_{2}\right\rangle=\sum_{\alpha \beta}(\Gamma \Delta)_{\alpha \beta}\left|e_{\alpha}\right\rangle \otimes\left|f_{\beta}\right\rangle$. In particular, if all the singular values are distinct, then two such density matrices are equivalent under local unitary transformation if and if they have exactly the same standard form.

We have investigated the equivalence under local unitary transformations for a class of rank-two bipartite mixed quantum states. A complete set of invariants has been presented 
such that any two of these states are locally equivalent if and only if all these invariants have equal values for these related density matrices.

Our method can be applied to another classification of quantum states, defined by local operations and classical communication (LOCC). Two states have the same kind of entanglement if they can be obtained from each other by LOCC with nonzero probability [14]. There have been many results for bipartite and multipartite pure states for their equivalence under SLOCC $[15,16,17]$. In [15] Dür et al showed that for pure three-qubit states there are six different classes of entanglement under SLOCC. Verstraete et al considered the entanglement of four-qubit case under SLOCC and concluded that there exist nine families of states corresponding to nine different ways of entanglement [16]. Nevertheless for mixed states few is known yet.

Corresponding to pure states, we say that $\rho_{1}$ and $\rho_{2}$ are equivalent under SLOCC if there exist invertible (but not necessarily unitary) matrices $P$ and $Q$ such that

$$
\rho_{2}=(P \otimes Q) \rho_{1}(P \otimes Q)^{\dagger}
$$

[Proposition] The density matrices $\rho_{1}$ and $\rho_{2}$, with $B_{1}$ and $B_{2}$ nonsingular, are equivalent under SLOCC if the following hold:

(i) $\operatorname{Tr}\left(\rho_{1}^{2}\right)=\operatorname{Tr}\left(\rho_{2}^{2}\right)$;

(ii) $\operatorname{Tr}\left(\left(A_{1} B_{1}^{-1}\right)^{\alpha}\right)=\operatorname{Tr}\left(\left(A_{2} B_{2}^{-1}\right)^{\alpha}\right), \forall \alpha=1, \ldots, m$;

(iii) $r\left(A_{1}\right)=r\left(A_{2}\right), r\left(B_{1}\right)=r\left(B_{2}\right), r\left(\left(B_{1}^{-1} A_{1}\right)^{\alpha}\right)=r\left(\left(B_{2}^{-1} A_{2}\right)^{\alpha}\right), \forall \alpha=1, \ldots, m$.

[Proof] From the conditions (ii) and (iii) we have that the pairs $\left(A_{1}, B_{1}^{-1}\right)$ and $\left(A_{2}, B_{2}^{-1}\right)$ are contragrediently equivalent. Hence there are invertible (but not necessarily unitary) matrices $S$ and $T$ such that $S A_{2}=A_{1} T, T B_{2}^{-1}=B_{1}^{-1} S$.

That is, we have $A_{2}=S^{-1} A_{1} T, \quad B_{2}=S^{-1} B_{1} T$. Accounting to the condition (i) which implies that the density matrices $\rho_{1}$ and $\rho_{2}$ have the same eigenvalues, the above relations give rise to the equivalence of $\rho_{1}$ and $\rho_{2}$ under SLOCC.

The classification of quantum states under local operations is of significance in quantum information processing. We have presented some criteria for the equivalence of some bipartite mixed states in arbitrary dimensions. Our results can be generalized to the case of multipartite states by considering bipartite decompositions. In terms of the method used in [11], our equivalence criteria for bipartite mixed states can be also used to study the equivalence of tripartite pure states. 


\section{References}

[1] M.A. Nielsen and I.L. Chuang, Quantum Computation and Quantum Information, Cambridge University Press, Cambridge, 2000.

[2] K. Chen, S. Albeverio and S.M. Fei, Phys. Rev. Lett. 95, 040504 (2005);

Phys. Rev. A 68, 062313 (2003).

[3] E.M. Rains, IEEE Transactions on Information Theory 46 54-59(2000).

[4] M. Grassl, M. Rötteler and T. Beth, Phys. Rev. A 58, 1833 (1998).

[5] Y. Makhlin, Quan. Inf. Pro. 1, 243 (2002).

[6] N. Linden, S. Popescu, and A. Sudbery, Phy. Rev. Lett. 83, 243 (1999).

[7] B.Z. Sun and S.M. Fei, Commun. Theor. Phys. 45, 1007-1010 (2006).

[8] S. Albeverio, S.M. Fei, P. Parashar, W.L. Yang, Phys. Rev. A 68, 010303 (2003).

[9] S. Albeverio, S.M. Fei and D. Goswami, Phys. Lett. A 340 37-42(2005).

[10] B.Z. Sun, S.M. Fei, X.Q. Li-Jost and Z.X. Wang, J. Phys. A 39 L43-L47(2006).

[11] S. Albeverio, L. Cattaneo, S.M. Fei and X.H. Wang, Int. J. Quant. Inform. 3 603-609 (2005); Rep. Math. Phys. 56 341-350 (2005).

[12] O. Holtz, Linear Algebra and its Applications, 310 11-17 (2000).

[13] W. Rudin, Functional Analysis, Tata Mcgraw-Hill Publishing Company Ltd., New Delhi (1974).

[14] C. H. Bennett, S. Popescu, D. Rohrlich, J. A. Smolin, A. V. Thapliyal, Phys. Rev. A 63 (2001) 012307.

[15] W. Dür, G. Vidal, J. I. Cirac, Phys. Rev. A 62, 062314(2000).

[16] F. Verstraete, J.Dehaene, B.De Moor and H. Verschelde Phys. Rev. A. 65, 052112 (2002).

[17] A. Acin, E. Jane, W. Dür and G. Vidal, Phys. Rev. Lett. 85, 4811 (2000).

H.J. Briegel and R. Raussendorf, Phy. Rev. Lett. 86, 910 (2001). 